\title{
DIGESTION OF KELOIDS, CICATRICES AND BUBOES IVITH PEPSIN-HYDROCHLORIC ACID
}

\author{
EDWARD AHLSWEDE, M.D. \\ Assistant Physician, University Skin Department, Eppendorf Hospital \\ (Professor Unna) \\ HAMBCRG, GERMANY
}

The latest histologic experiments of Unna have proved that the digestive power of pepsin and hydrochloric acid combined penetrates the horny layer of the epidermis and that this combination may even be used for carrying other chemical agents through the horny layer. A large number of chemicals can thus be caused to be absorbed by the surface of the skin; this has hitherto been impossible to accomplish, as, for instance, in the case of epinephrin. Naturally, in the first place it is the pepsin and hydrochloric acid itself which penetrate the intact horny layer. When this combination has penetrated to a certain depth it will affect certain diseases of the cutis and subcutis, as I shall try to show.

Our experiments were first tried on keloids and scarring after burns, cicatrices, with the intention of testing the possibility of digesting the fibrous (collagenous) tissue, of which these scars chiefly consist. The following solution was used: pepsin, 10 c.c.; muriatic acid, 1 c.c.; phenol, 1 c.c. Distilled water to make 200 c.c. was added. Phenol was added in order to prevent possible putrefaction of the decaying tissue.

The hydrochloric acid necessary for the digesting effect of pepsin has in itself some antiseptic property. We prefer, however, to add the same quantity of phenol, which in no way hinders digestion, but has the advantage of preventing the slightest degree of putrefaction. At the same time, phenol represents a useful antipruritic in many skin diseases. Compresses of absorbent cotton soaked in the above mentioned solution were applied and then covered with an impermeable cover in a large number of cases.

The cosmetic effect on scarring after burns was excellent. In many cases of fresh scars no trace was left after a systematic application of our digesting method. These successes led to the local treatment of ulcus durum, the latter being a reaction of the system against Spirochaeta pallida, chiefly by hypertrophy of the fibrous tissue, first surrounding the blood vessels.

The induration was digested by similar compresses with pepsinhydrochloric acid, with impermeable covering. Though the excision of the ulcer in this condition must be acknowledged as the quicker and more radical method, there are cases in which after a thorough arsphen- 
amin treatment the initial sclerosis has healed perfectly, yet a certain obstinate hardness remains, which will not give way to the strongest antisyphilitic treatment. Our pepsin compresses showed excellent success in those very obstinate cases in which the labia majora are changed into broad and hard masses of tissue.

From the same point of view we treated cases of adenitis caused by different infections, such as buboes following soft chancre. Almost every patient we treated showed a regression of the inflammation within from two to three weeks without an incision being necessary. In one case of bubo on both sides we made an incision in the left side, and injected iodoform glycerin, while we treated the right side with pepsinhydrochloric acid compresses. The latter was completely absorbed before the wound on the left side had healed. The effect of pepsinhydrochloric acid on infected glands in these cases can only be explained by the bactericidal power of phenol plus hydrochloric acid besides the digestion of the inflamed tissues by pepsin.

TREATMENT OF TUBERCULOUS GLANDS IN CHILDREN

We then took up the general treatment of all masses and packets of tuberculous glands in children, especially those of the throat and neck. In these cases, pepsin compresses represent a good substitute for surgical treatment, the more so as the incision of tuberculous glands. is not in all cases free from risk, since more than one case of lupus has had its origin in this procedure. 